\title{
PROPERTIES OF THE FOURIER ALGEBRA THAT ARE EQUIVALENT TO AMENABILITY
}

\author{
VIKTOR LOSERT
}

\begin{abstract}
It is shown that a locally compact group $G$ is amenable iff each multiplier on the Fourier algebra $A(G)$ is given by a function from the FourierStieltjes algebra $B(G)$. Another condition is that the norm of $A(G)$ is equivalent to that induced by the regular representation of $A(G)$.
\end{abstract}

Several properties of $A(G)$ have been shown to be equivalent to the amenability of $G$ : (1) $A(G)$ has a bounded approximate identity [Lep 2; H 1, Theorem 6, p. 120]. (2) $A(G)$ factorizes weakly, i.e. $A(G)$ is the linear span of $A(G) \cdot A(G)$ [Lo 1, Proposition 2, p. 138]. (3) $M(A(G), A(G))=B(G)$ ( $G$ discrete), where $M(\cdot)$ denotes the space of multipliers (see below). This was shown by Nebbia $[\mathbf{N}$, Theorem 2, p. 553]. In the case of groups having nonabelian free subgroups, the existence of multipliers on $A(G)$ not belonging to $B(G)$ was shown by FigàTalamanca and Picardello $[\mathbf{F P}]$. Some results on the case of Lie groups can be found in $[\mathbf{D H}]$. In this paper we extend (3) to nondiscrete groups.

THEOREM 1. The following statements are equivalent for any locally compact group $G$ :

(a) $G$ is amenable.

(b) $M(A(G), A(G))=B(G)$.

(c) The norm of $A(G)$ is equivalent to that induced by the regular representation of $A(G)$ (by multiplication on itself).

Condition (c) means there exists $c>0$ such that, for each $u \in A(G)$,

$$
\sup \left\{\|u v\|_{A}: v \in A(G),\|v\|_{A} \leq 1\right\} \geq c\|u\|_{A},
$$

or, equivalently (by the open mapping theorem), $A(G)$ is closed in $M(A(G), A(G))$. In the case of amenable groups, (c) (and the factorization property of $A(G)$ ) are consequences of the existence of a bounded approximate identity (and one even gets an isometry, i.e. $c=1$ in (c) and ordinary factorization). But for general Banach algebras, the reverse conclusion is not possible, as was shown by an example of Leinert [Lei].

Notations. $e$ denotes the unit of $G, \lambda$ a fixed Haar measure on $G$. In integrals, $d x, d y$, etc. refer to $\lambda$. For $f: G \rightarrow \mathbf{C}, x \in G$, we define left translation by $L_{x} f(y)=$ $f\left(x^{-1} y\right)$. Similarly, for $g \in L^{1}(G), f \in L^{p}(G)$, we use convolution to define $L_{g} f=g * f$. For $x \in G, \delta_{x}$ denotes the Dirac measure concentrated at $x$. $\mathcal{K}(G)$ denotes the space of continuous (complex-valued) functions $f$ on $G$ with compact support supp $f . C_{\mathrm{lu}}(G)$ denotes the space of left-uniformly continuous functions; it

Received by the editors September 20, 1983.

1980 Mathematics Subject Classification. Primary 43A07; Secondary 43A22, 43A35, 43A30.

Key words and phrases. Locally compact groups, amenability, Fourier algebra, multipliers. 
consists of those bounded functions $f: G \rightarrow \mathbf{C}$ for which $x \rightarrow L_{x} f$ is continuous with respect to \|\|$_{\infty}$. For the definition and simple properties of means on $L^{\infty}(G)$ and $C_{\mathrm{lu}}(G)$, we refer to $[\mathbf{G r}] . \quad G$ is called amenable if there exists a left-invariant mean on $L^{\infty}(G)$.

For the definition and properties of the Fourier algebra $\left(A(G),\|\|_{A}\right)$ and the Fourier-Stieltjes algebra $B(G)$, we refer to $[\mathbf{E}] . P_{1}(G)$ denotes the set of continuous positive-definite functions $u: G \rightarrow \mathbf{C}$ such that $u(e)=1$. By [E, p. 218], any $u \in A(G)$ can be written as $u(x)=\left(L_{x} h, k\right)$, where $h, k \in L^{2}(G)$ and ( , ) denotes the inner product of $L^{2}(G)$. If, in addition, $u \in P_{1}(G)$, one can assume that $h=k$ and $\|h\|_{2}=1\left[\mathbf{E}\right.$, p. 188]. $\mathrm{VN}(G)$ denotes the von Neumann algebra on $L^{2}(G)$ generated by the operators $L_{x}(x \in G), C_{L}^{*}(G)$ denotes the $C^{*}$-algebra generated by all $L_{f}\left(f \in L^{1}(G)\right)$, i.e. the norm closure in the space of operators. By $[\mathbf{E}, \mathbf{p}$. 210], $\mathrm{VN}(G)$ can be identified with the dual of $A(G)$, for $T \in \mathrm{VN}(G), u \in A(G)$ as above, the duality is given by $\langle T, u\rangle=(T h, k)$.

A linear operator $\Gamma: A(G) \rightarrow A(G)$ is called an $(A(G)$-) multiplier if $\Gamma(u v)=$ $u \Gamma(v)$ for all $u, v \in A(G)$ (similarly for arbitrary $A(G)$-modules). The space of multipliers is denoted by $M(A(G), A(G))$. It follows easily from [E, (3.34), p. 222] and the closed graph theorem that any $\Gamma \in M(A(G), A(G))$ is norm bounded and given by a multiplication operator $\Gamma(u)=g u$, where $g: G \rightarrow \mathbf{C}$ is bounded and continuous. Conversely, any $g \in B(G)$ defines a multiplier [E, (3.4), p. 208], i.e. $B(G) \subseteq M(A(G), A(G))$.

LEMMA 1. If $T \in C_{L}^{*}(G)$ and $K$ is a compact subset of $G$, then $T \mid L^{2}(K)$ is compact. (In particular, $C_{L}^{*}(G)$ has a unit iff $G$ is discrete.)

Proof. $T \mid L^{2}(K)$ denotes the restriction of $T$ considered as a mapping from $L^{2}(K)$ (subspace of $L^{2}(G)$ ) to $L^{2}(G)$. We may assume $T=L_{f}$, with $f \in \mathcal{K}(G)$, and $\lambda(K)>0$. If $g \in L^{2}(K)$ with $\|g\|_{2} \leq \lambda(K)^{-1 / 2}$, then $\|g\|_{1} \leq 1$; hence $L_{f}(g)=f * g=\int_{K} g(x) f * \delta_{x} d x$ belongs to the closed, absolutely convex hull of $\left\{f * \delta_{x}: x \in K\right\}$. (If $L_{f}$ is invertible, this is possible only if $L^{2}(K)$ is finite dimensional.)

REMARK. Even if $G$ is not discrete, it may happen that the spectrum of $C_{L}^{*}(G)$ (or $C^{*}(G)$ ) is compact (see [B, p. 144]).

In Lemma 2 and Proposition $1, f$ denotes a continuous function on $\mathbf{R}$ such that $0 \leq f \leq 1, f(t)=0$ for $t \leq 1 / 4$ and $f(t)=1$ for $t \geq 1 / 2$.

LEMmA 2. (a) If $R \in \mathrm{VN}(G), R \leq 1, u \in A(G) \cap P_{1}(G), x \in G,\langle R, u\rangle>1-$ $\delta^{2} / 2,\left\langle L_{x^{-1}} R L_{x}, u\right\rangle>1-\delta^{2} / 2, R^{\prime}=f(R), S=R^{\prime} L_{x} R^{\prime}$, then $\left\langle S, L_{x} u\right\rangle>1-2 \delta$.

(b) If $R, u$ are as in (a), $R_{i} \in \mathrm{VN}(G)$ commute pairwise, $0 \leq R_{i} \leq 1$ (i= $1, \ldots, n),\left\langle\sum_{i=1}^{n} R_{i}^{2}, u\right\rangle<\left(\delta^{2} / 4 n\right)^{2}$, and $R^{\prime \prime}=\prod_{i=1}^{n}\left(1-R_{i}\right) R \prod_{i=1}^{n}\left(1-R_{i}\right)$, then $\left\langle R^{\prime \prime}, u\right\rangle>1-\delta^{2}$.

ProOF. (a) We have $u(x)=\left(L_{x} h, h\right)$ for some $h \in L^{2}(G)$ with $\|h\|_{2}=1$. Since $\left(1-R^{\prime}\right)^{2} \leq 2(1-R)$, we get

$$
\left\|h-R^{\prime} h\right\|_{2}^{2} \leq 2((1-R) h, h)=2(1-\langle R, u\rangle)<\delta^{2}
$$

and, similarly, $\left\|L_{x} h-R^{\prime} L_{x} h\right\|_{2}^{2}<\delta^{2}$. Now

$$
\left\langle S, L_{x} u\right\rangle=\left(S h, L_{x} h\right)=\left(L_{x} R^{\prime} h, R^{\prime} L_{x} h\right)>\left(L_{x} h, L_{x} h\right)-2 \delta=1-2 \delta .
$$


(b) Keeping the notation of (a), we have $\left\|R_{i} h\right\|_{2}<\delta^{2} / 4 n$. Since

$$
1-\prod_{i=1}^{n}\left(1-R_{i}\right)=\sum_{i=1}^{n} R_{i} \prod_{j=1}^{i-1}\left(1-R_{j}\right)
$$

we get $\left\|\prod_{i=1}^{n}\left(1-R_{i}\right) h-h\right\|_{2}<\delta^{2} / 4$ and the result follows.

In the case of a nondiscrete abelian group $G$, multiplication by characters defines an isometry on $A(G)$ and, given $u_{1}, u_{2} \in A(G)$, one can find $\chi_{1}, \chi_{2} \in G^{-}$such that $\left(u_{1} \chi_{1}\right)$ and $\left(u_{2} \chi_{2}\right)$ ^ have "almost" disjoint supports. The following proposition shows that similar things can be done for general $G$, at least for translates of positive-definite functions.

Proposition 1. Assume that $G$ is not discrete, $u_{i} \in A(G) \cap P_{1}(G), x_{i} \in G(i=$ $1, \ldots, n), \varepsilon>0$. Then there exist $w_{i} \in P_{1}(G), S_{i} \in C_{L}^{*}(G)$ satisfying: $\left\|S_{i}\right\| \leq 1$, the images of $S_{i}\left(\right.$ resp. $\left.S_{i}^{*}\right)$ are pairwise orthogonal, $\left\langle S_{i}, L_{x_{i}}\left(u_{i} w_{i}\right)\right\rangle>1-\varepsilon$ for all $i$.

In particular,

$$
\left\|\sum_{i=1}^{n} \mu_{i} L_{x_{i}}\left(u_{i} w_{i}\right)\right\|_{A}>(1-\varepsilon) \sum_{i=1}^{n}\left|\mu_{i}\right| \quad \text { for all } \mu_{i} \in \mathbf{C} .
$$

Proof. We use induction on $n$. Observe that $C_{L}^{*}(G)$ is $\mathrm{w}^{*}$-dense in $\mathrm{VN}(G)[\mathbf{D i}$ 1, I.3.4, Corollary 1, p. 45] and that the same is true for the hermitian parts of the unit balls [Di 1, I.3.5, Theorem 3, p. 47]. For $n=1$ take $R \in C_{L}^{*}(G)$ such that $R \leq 1,\left\langle R, u_{1}\right\rangle>1-\varepsilon^{2} / 8$ and $\left\langle L_{x_{1}^{-1}} R L_{x_{1}}, u_{1}\right\rangle>1-\varepsilon^{2} / 8$. Put $R_{1}=f(R), \bar{R}_{1}=$ $f(2 R), S_{1}=R_{1} L_{x_{1}} R_{1}$. Then by Lemma $2\left(\right.$ a) $\left\langle S_{1}, L_{x_{1}} u_{1}\right\rangle>1-\varepsilon$. Put $w_{1}=1$.

Now assume we have constructed $S_{i}$ and $w_{i}(i=1, \ldots, n)$ as needed, so that $S_{i}=R_{i} L_{x_{i}} R_{i}, R_{i}, \bar{R}_{i} \in C_{L}^{*}(G), 0 \leq R_{i}, \bar{R}_{i} \leq 1, \bar{R}_{i} \bar{R}_{j}=0$ for $i \neq j$ and $R_{i} \bar{R}_{i}=R_{i}$. Put $T_{1}=\sum_{i=1}^{n} \bar{R}_{i}^{2}, T_{2}=L_{x_{n+1}^{-1}} T_{1} L_{x_{n+1}}$. Then $u_{n+1} T_{k} \in C_{L}^{*}(G)$ [E, p. 224]. By Lemma 1 there exists $h \in L^{2}(G)$ such that $\|h\|_{2}=1$ and $\left(\left(u_{n+1} T_{k}\right) h, h\right)<$ $\left(\varepsilon^{2} / 64 n\right)^{2}$ for $k=1,2$. Put $w_{n+1}(x)=\left(L_{x} h, h\right)$. Then $\left\langle T_{k}, u_{n+1} w_{n+1}\right\rangle<\left(\varepsilon^{2} / 64 n\right)^{2}$.

Now, as above, choose $R \in C_{L}^{*}(G)$ such that $R \leq 1,\left\langle R, u_{n+1} w_{n+1}\right\rangle>1-$ $\varepsilon^{2} / 16,\left\langle L_{x_{n+1}^{-1}} R L_{x_{n+1}}, u_{n+1} w_{n+1}\right\rangle>1-\varepsilon^{2} / 16$. Put

$$
R^{\prime}=\prod_{i=1}^{n}\left(1-\bar{R}_{i}\right) R \prod_{i=1}^{n}\left(1-\bar{R}_{i}\right), \quad R_{n+1}=f\left(R^{\prime}\right), \quad \bar{R}_{n+1}=f\left(2 R^{\prime}\right) .
$$

Then $R^{\prime} R_{i}=0$ for $i=1, \ldots, n$. Using Lemma 2(b) and 2(a) and replacing $\bar{R}_{i}$ by $\left(1-f\left(4 R^{\prime}\right)\right) \bar{R}_{i}\left(1-f\left(4 R^{\prime}\right)\right)$ for $i=1, \ldots, n$, we get all properties needed for the induction step.

LEMMA 3. Assume that $G$ is not discrete and $\sup \left\{\|u v\|_{A}:\|v\|_{A} \leq 1\right\}>c\|u\|_{A}$ for all $u \in A(G)$. Then, given $u \in A(G) \cap P_{1}(G), x_{1}, \ldots, x_{n} \in G$, there exists $v \in A(G) \cap P_{1}(G)$ such that $\sum_{i=1}^{n}\left\|v \cdot L_{x_{i}} u\right\|_{A}>c n / 4$.

Proof. By Proposition 1 there exists $w_{i} \in P_{1}(G)$ such that $\left\|\sum_{i=1}^{n} L_{x_{i}}\left(u w_{i}\right)\right\|_{A}$ $>n / 2$. By assumption there exists $v \in A(G)$ with $\|v\|_{A} \leq 1$ such that

$$
\left\|v \cdot \sum_{i=1}^{n} L_{x_{i}}\left(u w_{i}\right)\right\|_{A}>\frac{c n}{2}
$$


Replacing $c n / 2$ by $c n / 4$, we may assume that $v$ is hermitian. Then by [E, (3.15), p. 212 and $(2.7)$, p. 193] we get $v$ positive-definite. Now the result follows.

The next step uses a device due to H. Rindler (see [Lo 1, Lemma 4, p. 136]).

LEMmA 4. For $a, b \in L^{2}(G)$ with $a, b \geq 0$, we have

$$
\int_{G}\left|a^{2}-b^{2}\right| d \lambda \leq\left(\left(\int_{G} a^{2}+b^{2} d \lambda\right)^{2}-4\left(\int_{G} a b d \lambda\right)^{2}\right)^{1 / 2} .
$$

LEMMA 5. Let $0<\varepsilon<1, U, V, W$ be e-neighbourhoods such that $U W \subseteq$ $V, \lambda(V)<(1+\varepsilon) \lambda(W)$. Let $h, k \in L^{2}(G), T \in \mathrm{VN}(G)$ be such that $\|h\|_{2} \leq$ 1, $\|k\|_{2} \leq 1,\|T\| \leq 1, \operatorname{supp} T \subseteq U$. Define a mean $M$ on $C_{\mathrm{lu}}(G)$ by $M(f)=$ $\int_{G} f \cdot|h|^{2} d \lambda$. Then for $x \in G, f \in C_{\mathrm{lu}}(G)$,

$$
\begin{aligned}
\left|M\left(L_{x} f-f\right)\right| \leq & 2 \sup \left\{\left\|L_{y} f-f\right\|_{\infty}: y \in V\right\} \\
& +\|f\|_{\infty}\left(2+3 \varepsilon-\left|\left(T(h \otimes k), L_{x^{-1}} h \otimes k\right)\right|^{2}\right) .
\end{aligned}
$$

(See $\left[\mathbf{E}, 4.5\right.$, p. 226] for the definition of $\operatorname{supp} T$; the representation $x \rightarrow L_{x} \otimes L_{x}$ is quasi-equivalent to $L$ [D 2, Example 13.11.3, p. 306], hence $T$ is also defined on $L^{2}(G) \otimes L^{2}(G)[$ D 2, 5.3.1, p. 118].)

ProOF. Put $\delta=\sup \left\{\left\|L_{y} f-f\right\|_{\infty}: y \in V\right\}$. Since $\|k\|_{2}=1$, we have

$$
\begin{gathered}
M(f)=\int_{G \times G} f(y)|h(y) k(z)|^{2} d(y, z) . \\
\left.\left|\int_{G \times G} f(y)\right| h \otimes k\right|^{2}(y, z) d(y, z) \\
-\lambda(V)^{-1} \int_{V} \int_{G \times G} f\left(t^{-1} y\right)|h \otimes k|^{2}(y, z) d(y, z) d t \mid<\delta
\end{gathered}
$$

and the second integral equals

$$
\lambda(V)^{-1} \int_{G} f(y) \int_{V \times G}|h \otimes k|^{2}(t y, z) d(t, z) d y .
$$

Similarly,

$$
\left.\left|M\left(L_{x} f\right)-\lambda(W)^{-1} \int_{G} f(y) \int_{W \times G}\right| L_{x^{-1}} h \otimes k\right|^{2}(t y, z) d(t, z) d y \mid<\delta .
$$

Since $\left|\lambda(V)^{-1}-\lambda(W)^{-1}\right|<\varepsilon \lambda(V)^{-1}$, we get (putting

$$
a(y)^{2}=\int_{W \times G}\left|L_{x^{-1}} h \otimes k\right|^{2}(t y, z) d(t, z)
$$

and

$$
\begin{gathered}
\left.b(y)^{2}=\int_{V \times G}|h \otimes k|^{2}(t y, z) d(t, z)\right) \\
\left|M\left(L_{x} f-f\right)\right| \leq 2 \delta+\|f\|_{\infty}\left(\varepsilon+\lambda(W)^{-1}\right) \int_{G}\left|a(y)^{2}-b(y)^{2}\right| d y .
\end{gathered}
$$

Now we apply Lemma 4 . We have $\int_{G} a(y)^{2} d y=\lambda(W), \int_{G} b(y)^{2} d y=\lambda(V)$. Furthermore, $a(y)=\Delta(y)^{-1 / 2}\left\|L_{x^{-1}} h \otimes k \mid W y \times G\right\|_{2} \quad$ and $\quad b(y)=\Delta(y)^{-1 / 2}\|h \otimes k \mid V y \times G\|_{2}$. ( $\Delta$ denotes the Haar modulus of $G$.) 
By our assumptions on $T$, we have

$$
\left\|h \otimes k\left|V y \times G\left\|_{2} \geq\right\| T(h \otimes k)\right| V y \times G\right\|_{2} \geq\|T(h \otimes k) \mid W y \times G\|_{2} .
$$

(It follows easily from $[\mathbf{E}, 4.8$, p. 226] that $\operatorname{supp}(T(h \otimes k)) \subseteq \operatorname{supp} T \cdot \operatorname{supp}(h \otimes k)$, where - refers to the diagonal action of $G$ on $G \times G$.) This gives

$$
\begin{aligned}
\int_{G} a(y) b(y) d y & \geq \int_{G}\left\|L_{x^{-1}} h \otimes k\left|W y \times G\left\|_{2}\right\| T(h \otimes k)\right| W y \times G\right\|_{2} \Delta\left(y^{-1}\right) d y \\
& \geq \int_{G} \int_{W y \times G}\left|\left(L_{x^{-1}} h \otimes k\right) \cdot T(h \otimes k)\right|(t, z) d(t, z) \Delta\left(y^{-1}\right) d y \\
& =\int_{G} \int_{W \times G}\left|\left(L_{x^{-1}} h \otimes k\right) \cdot T(h \otimes k)\right|(t y, z) d(t, z) d y \\
& \geq \lambda(W)\left|\left(T(h \otimes k), L_{x^{-1}} h \otimes k\right)\right| .
\end{aligned}
$$

Hence, by Lemma 4 ,

$$
\begin{aligned}
\int_{G}\left|a(y)^{2}-b(y)^{2}\right| d y & \leq\left((\lambda(W)+\lambda(V))^{2}-4 \lambda(W)^{2}\left|\left(T(h \otimes k), L_{x^{-1}} h \otimes k\right)\right|^{2}\right)^{1 / 2} \\
& \leq 2 \lambda(W)\left(1+2 \varepsilon-\mid\left(T(h \otimes k),\left.L_{x^{-1}}(h \otimes k)\right|^{2}\right)^{1 / 2}\right. \\
& \leq \lambda(W)\left(2+2 \varepsilon-\left|\left(T(h \otimes k), L_{x^{-1}} h \otimes k\right)\right|^{2}\right)
\end{aligned}
$$

and the result follows.

If $M$ is a mean on $L^{\infty}(G)$ or $C_{\mathrm{lu}}(G), x \in G$, we write

$$
d(M, x)=\sup \left\{\left|M\left(L_{x} f-f\right)\right|:\|f\|_{\infty} \leq 1\right\} .
$$

Proposition 2. Assume there exists $c<2$ such that for each $x_{1}, \ldots, x_{n} \in G$ (not necessarily distinct points) there exists a mean $M$ on $C_{\mathrm{lu}}(G)$ with $\sum_{i=1}^{n} d\left(M, x_{i}\right)$ $\leq c n$. Then $G$ is amenable.

Proof. This will be done in two steps. Let $\mathcal{M}$ be the set of means on $C_{\mathrm{lu}}(G)$. First we show that, given $x_{1}, \ldots, x_{n} \in G$, there exists $M \in \mathcal{M}$ such that $d\left(M, x_{i}\right) \leq c$ for $i=1, \ldots, n$.

To each $M \in \mathcal{M}$ we associate the $n$-tuple $\left(d\left(M, x_{i}\right)\right)_{i=1}^{n} \in \mathbf{R}^{n}$. Let $C$ be the convex hull of these elements, take $\varepsilon>0$ and assume that $C$ contains no vector $\left(t_{i}\right)$ with $\left\|\left(t_{i}\right)\right\|_{\infty} \leq c+\varepsilon$. Then we apply the separation theorem for convex sets. There exists $\left(u_{i}\right) \in \mathbf{R}^{n}$ such that $\left\|\left(u_{i}\right)\right\|_{1}=1$ and $\sum_{i=1}^{n} t_{i} u_{i} \geq c+\varepsilon$ for all $\left(t_{i}\right) \in C$. We may assume that $u_{i} \geq 0$ and $u_{i}=p_{i} / q \in \mathbf{Q}$ for $i=1, \ldots, n$ (replacing $\varepsilon$ by $\varepsilon / 2)$. Then $\sum p_{i}=q$. Put $y_{1}=\cdots=y_{p_{1}}=x_{1}, y_{p_{1}+1}=\cdots=y_{p_{1}+p_{2}}=x_{2}$, etc. Then, by assumption, there exists $M \in \mathcal{M}$ such that $\sum_{j=1}^{q} d\left(M, y_{j}\right) \leq c q$. But

$$
q^{-1} \sum_{j=1}^{q} d\left(M, y_{j}\right)=q^{-1} \sum_{i=1}^{n} p_{i} d\left(M, x_{i}\right)=\sum_{i=1}^{n} u_{i} d\left(M, x_{i}\right) \geq c+\frac{\varepsilon}{2}
$$

by definition of $\left(u_{i}\right)$, and we arrive at a contradiction. Thus, there exists $\left(t_{i}\right) \in C$ with $\left\|\left(t_{i}\right)\right\|_{\infty} \leq c+\varepsilon$. Then $t_{i}=\sum \lambda_{k} d\left(M_{k}, x_{i}\right)$ for some $M_{k} \in \mathcal{M}, \lambda_{k} \geq 0$ with $\sum \lambda_{k}=1$. Put $M=\sum \lambda_{k} M_{k}$. Then $M \in \mathcal{M}$ and $d\left(M, x_{i}\right) \leq t_{i} \leq c+\varepsilon$ for all $i$. Since $\varepsilon>0$ was arbitrary, the first step follows from the compactness of $\mathcal{M}$. 
The second step is now to show that, given $x_{1}, \ldots, x_{n} \in G$, there exists a mean $M$ on $L^{\infty}(G)$ such that $d\left(M, x_{i}\right) \leq c$ for $i=1, \ldots, n$. Then the result will follow from [Lo1, Lemma 3 and Theorem 1 (see also [Gi, Lep 1]). The extension from $C_{\mathrm{lu}}(G)$ to $L^{\infty}(G)$ is done essentially as in [Gr, p. 28]. Fix $u \in L^{1}(G)$ with $u \geq 0,\|u\|_{1}=1$. For $M \in \mathcal{M}, f \in L^{\infty}(G)$, put $M_{1}(f)=M(u * f)$. Then $M_{1}$ is a mean on $L^{\infty}(G)$.

Given $x_{1}, \ldots, x_{n} \in G$, we have $M_{1}\left(L_{x_{i}} f-f\right)=M\left(\left(u * \delta_{x_{i}}-u\right) * f\right)$. For $\varepsilon>0$ there exists $v \in L^{1}(G)$ with $v \geq 0,\|v\|_{1}=1$, such that $\left\|\left(u * \delta_{x_{i}}-u\right) *\left(v-\delta_{e}\right)\right\|_{1}<\varepsilon$ for $i=1, \ldots, n$. Then we can find $y_{j} \in G, \lambda_{j} \geq 0$ with $\sum \lambda_{j}=1$ such that $\left\|\left(u-\sum \lambda_{j} \delta_{y_{j}}\right) *\left(\delta_{x_{i}} * v-v\right)\right\|_{1}<\varepsilon$. This gives combined (assuming $\left.\|f\|_{\infty} \leq 1\right)$ :

$$
\begin{aligned}
\left|M_{1}\left(L_{x_{i}} f-f\right)\right| & <2 \varepsilon+\sum \lambda_{j}\left|M\left(\delta_{y_{j}} *\left(\delta_{x_{i}} * v-v\right) * f\right)\right| \\
& =2 \varepsilon+\sum \lambda_{j}\left|M\left(\left(\delta_{y_{j} x_{i} y_{j}^{-1}}-\delta_{e}\right) * \delta_{y_{j}} * v * f\right)\right| \\
& \leq 2 \varepsilon+\sum \lambda_{j} d\left(M, y_{j} x_{i} y_{j}^{-1}\right) .
\end{aligned}
$$

Choosing $M \in \mathcal{M}$ so that $d\left(M, y_{j} x_{i} y_{j}^{-1}\right) \leq c$ for all $i, j$, we get $d\left(M_{1}, x_{i}\right) \leq c+2 \varepsilon$ for all $i$.

Proof of TheOREM 1. If $G$ is amenable, then $(\mathrm{a}) \Rightarrow(\mathrm{b})$ by [De, Theorem 9; $\mathbf{H ~ 2 , ~ T h e o r e m ~ 1 ; ~ o r ~} \mathbf{R}$, Theorem 1]. (b) $\Rightarrow(\mathrm{c})$ is trivial. Now assume that $\sup \left\{\|u v\|_{A}:\|v\|_{A} \leq 1\right\}>c\|u\|_{A}$ for each $u \in A(G)$ (where $c>0$ ). The discrete case can be settled as in [N]: Take $h \in l^{2}(G)$. By Lemma 2 of $[\mathbf{N}]$ there is $\Phi \in l^{\infty}(G)$ such that $\|\Phi\|_{\infty}=1$ and $\|h \Phi\|_{A} \geq c_{1}\|h\|_{2}$. By assumption there is $u \in A(G)$ such that $\|u\|_{A}=1$ and $\|u h \Phi\|_{A} \geq c\|h \Phi\|_{A} \geq c c_{1}\|h\|_{2}$. But $\|u h \Phi\|_{A} \leq\|u h \Phi\|_{2} \leq\|u h\|_{2}$. $c, c_{2}$ are independent of $u, \Phi$ and therefore $l^{2}(G)$ is closed in $M\left(A(G), l^{2}(G)\right)$. Now the same proof as for Theorem 1 of $[\mathbf{N}]$ shows that $G$ is amenable.

Now assume $G$ is nondiscrete. Take $x_{1}, \ldots, x_{n} \in G$ and put $\varepsilon=c^{2} / 200$. If $V$ is a neighbourhood of $e$, choose neighbourhoods $U, W$ such that $\lambda(W)>(1+\varepsilon)^{-1} \lambda(V)$ and $U W \subseteq V$. Take $u \in A(G) \cap P_{1}(G)$ with supp $u \in U$. By Lemma 3 there exists $v \in A(G) \cap P_{1}(G)$ such that

$$
\sum_{i=1}^{n}\left\|v \cdot L_{x_{i}} u\right\|_{A}>\frac{c n}{4}
$$

Put $c_{i}=\left\|v \cdot L_{x_{i}} u\right\|_{A}, v(x)=\left(L_{x} h, h\right), u(x)=\left(L_{x} k, k\right)$, where $h, k \in L^{2}(G),\|h\|_{2}$ $=\|k\|_{2}=1$. Then there exists $T_{i}^{\prime} \in \operatorname{VN}(G)$ such that $\left\|T_{i}^{\prime}\right\| \leq 1$ and $\left\langle T_{i}^{\prime}, v \cdot L_{x_{i}} u\right\rangle=$ $c_{i}$. We have $\left\langle L_{x_{i}^{-1}} T_{i}^{\prime}, L_{x_{i}^{-1}} v \cdot u\right\rangle=\left\langle T_{i}^{\prime}, v \cdot L_{x_{i}} u\right\rangle$. Put $w(x)=\lambda(W)^{-1} \lambda(x W \cap V)$. Then $w=1$ on $U$ and $\|w\|_{A} \leq(\lambda(V) / \lambda(W))^{1 / 2} \leq 1+\varepsilon$. Put $T_{i}=w \cdot\left(L_{x_{i}^{-1}} T_{i}^{\prime}\right)$. $(1+\varepsilon)^{-1}$. Then $\left\|T_{i}\right\| \leq 1$,

$$
\left(T_{i}(h \otimes k), L_{x_{i}^{-1}} h \otimes k\right)=\left\langle T_{i}, L_{x_{i}^{-1}} v \cdot u\right\rangle=c_{i}(1+\varepsilon)^{-1} \geq c_{i} / 2
$$

(since $\operatorname{supp} u \subseteq U$ ). We define a mean $M$ on $C_{\mathrm{lu}}(G)$ by $M(f)=\int_{G} f(x)|h(x)|^{2} d x$. By Lemma 5 ,

$$
\left|M\left(L_{x_{i}} f-f\right)\right| \leq 2 \sup \left\{\left\|L_{y} f-f\right\|: y \in V\right\}+\|f\|_{\infty}\left(2+3 \varepsilon-c_{i}^{2} / 4\right) .
$$

We have $\sum c_{i} \geq n c / 4$, hence $\sum c_{i}^{2} \geq n(c / 4)^{2}$. Thus

$$
\sum_{i=1}^{n}\left|M\left(L_{x_{i}} f-f\right)\right| \leq 2 \sup \left\{\left\|L_{y} f-f\right\|: y \in V\right\}+n\|f\|_{\infty}\left(2+3 \varepsilon-\frac{c^{2}}{64}\right) .
$$


The neighbourhood $V$ was arbitrary. Considering a limit along the neighbourhood filter, we get a mean $M$ on $C_{\mathrm{lu}}(G)$ such that $\sum_{i=1}^{n} d\left(M, x_{i}\right) \leq n\left(2+3 \varepsilon-c^{2} / 64\right)$. Now Proposition 2 shows that $G$ is amenable.

A related criterion for amenability can be obtained using the space $S_{0}(G)$ (see $[\mathbf{F e}])$. It can be defined as follows: Let $\left(\psi_{i}\right)$ be a bounded uniform partition of unity in $A(G)$, i.e. all $\psi_{i}$ are uniformly bounded in $A(G)$, supp $\psi_{i}$ is contained in a left-translate of a fixed compact subset $K$ and there exists $n_{0}$ such that for each $x \in G$ the supports of at most $n_{0}$ functions $\psi_{i}$ intersect $x K$ (e.g. for $G=\mathbf{R}$ one can take trapezoidal functional of width 2 with integer endpoints; see also [Lo 2 , Proposition 1, p. 131]). Then $S_{0}(G)=\left\{u: G \rightarrow \mathbf{C}:\|u\|_{S_{0}}=\sum\left\|u \psi_{i}\right\|_{A}<\infty\right\}$.

THEOREM 2. $G$ is amenable iff $M\left(A(G), S_{0}(G)\right)=S_{0}(G)$.

Proof. Let $G$ be amenable and consider $\Phi \in M\left(A(G), S_{0}(G)\right)$. Given finitely many $\psi_{i}\left(i \in I_{0}\right), \varepsilon>0$, there exists $u \in A(G)$ such that $\|u\|_{A}<1+\varepsilon, u(x)=1$ for $x \in \operatorname{supp} \psi_{i}\left(i \in I_{0}\right)$. Hence $u \Phi \in S_{0}(G)$ and $\sum_{i \in I_{0}}\left\|\Phi \psi_{i}\right\|_{A}=\sum_{i \in I_{0}}\left\|u \Phi \psi_{i}\right\|_{A} \leq$ $\|\Phi\|_{M}(1+\varepsilon)$ (where \|\|$_{M}$ denotes the multiplier norm). Hence $\Phi \in S_{0}(G)$.

Conversely, assume that $\sup \left\{\|u \Phi\|_{S_{0}}:\|u\|_{A} \leq 1\right\}>c\|\Phi\|_{S_{0}}$ for all $\Phi \in S_{0}(G)$. Then we proceed as in Theorem 1 . Choose $u \in A(G) \cap P_{1}(G)$ and take $x_{1}, \ldots, x_{n} \in$ $G$. As in Lemma 3, there is $v \in S_{0}(G)$, such that $\sum_{j}\left\|v \cdot L_{x_{j}} u\right\|_{S_{0}}>c n / 2,\|v\|_{S_{0}} \leq 1$. Since $S_{0}(G)$ is contained in $A(G)$, we have $\|v\|_{A} \leq c_{1}$. For functions whose support is contained in a translate of a fixed compact set, the $A$ - and $S_{0}$-norms are equivalent $\left[\mathbf{F e}\right.$, p. 275]. Thus we get $\sum_{j}\left\|v \cdot L_{x_{j}} u\right\|_{A}>c c_{2} n / 2$. Now continue as in Lemma 3 and Theorem 1 . In the discrete case, $S_{0}(G)=l^{1}(G)$, hence one can use $[\mathbf{N}$, Theorem 1].

\section{REFERENCES}

[B] L. Baggett, A separable group having a discrete dual is compact, J. Funct. Anal. 10 (1972), 131-148.

[De] A. Derighetti, Some results on the Fourier-Stieltjes algebra of a locally compact group, Comment. Math. Helv. 45 (1970), 219-228.

[DH] J. De Canniére and U. Haagerup, Multipliers of the Fourier algebras of some simple Lie groups and their discrete subgroups, Amer. J. Math. (to appear).

[Di 1] J. Dixmier, Von Neumann algebras, North-Holland, Amsterdam, 1981.

[Di 2] _,$C^{*}$-algebras, North-Holland, Amsterdam, 1977.

[E] P. Eymard, L'algèbre de Fourier d'un groupe localement compact, Bull. Soc. Math. France 92 (1964), 181-236.

[Fe] H. G. Feichtinger, On a new Segal algebra, Monatsh. Math. 92 (1981), 269-289.

[FP] A. Figà-Talamanca and M. A. Picardello, Multiplicateurs de $A(G)$ qui ne sont pas dans $B(G)$, C.R. Acad. Sci. Paris Sér. A-B 277 (1973), A117-A119.

[Gi] J. E. Gilbert, Convolution operators on $L^{p}(G)$ and properties of locally compact groups, Pacific J. Math. 24 (1968), 257-268.

[Gr] F. P. Greenleaf, Invariant means on topological groups and their applications, Van Nostrand, New York, 1969.

[H 1] C. Herz, Harmonic synthesis for subgroups, Ann. Inst. Fourier (Grenoble) 23 (1973), 91-123.

[H 2] __ Une généralization de la notion de transformée de Fourier-Stieltjes, Ann. Inst. Fourier (Grenoble) 24 (1974), 145-157.

[Lei] M. Leinert, A factorable Banach algebra with inequivalent regular representation norm, Proc. Amer. Math. Soc. 60 (1976), 161-162.

[Lep 1] H. Leptin, On locally compact groups with invariant means, Proc. Amer. Math. Soc. 19 (1968), 489-494.

[Lep 2] _ Sur l'algèbre de Fourier d'un groupe localement compact, C.R. Acad. Sci. Paris Sér. A-B 266 (1968), A1180-A1182. 
[Lo 1] V. Losert, Some properties of groups without the property $P_{1}$, Comment. Math. Helv. 54 (1979), 133-139.

[Lo 2] _ , A characterization of the minimal strongly character invariant Segal algebra, Ann. Inst. Fourier (Grenoble) 30 (1980), 129-139.

[N] C. Nebbia, Multipliers and asymptotic behaviour of the Fourier algebra of nonamenable groups, Proc. Amer. Math. Soc. 84 (1982), 549-554.

[R] P. F. Renaud, Centralizers of the Fourier algebra of an amenable group, Proc. Amer. Math. Soc. 32 (1972), 539-542.

INSTITUT FÜr MATHEMATIK, UNIVERSität WiEN, STRUdlhofgasse 4, A-1090 WiEN, Austria 